\title{
The Effect of Anesthesia Depth Monitoring on Postoperative Delirium and Postoperative Cognitive Dysfunction in Non-cardiac Surgery Patients-A Systematic Review and Meta-Analysis
}

Ping Huang ( $\nabla$ huangpingle@126.com )

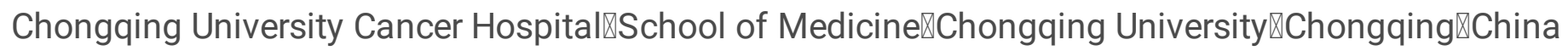
https://orcid.org/0000-0001-5454-0764

\section{Shufang Sun}

Chongqing University Cancer Hospital,School of Medicine,Chongqing University,Chongqing,China Hongliang Liu

Chongqing University Cancer Hospital,School of Medicine,Chongqing University,Chongqing,China

\section{Research}

Keywords: Bispectral Index Monitoring, Auditory Evoked Potentials, Primitive EEG Monitoring

Posted Date: May 6th, 2021

DOI: https://doi.org/10.21203/rs.3.rs-439899/v1

License: (c) (1) This work is licensed under a Creative Commons Attribution 4.0 International License.

Read Full License 


\section{Abstract}

Objective: To systematically evaluate the influence of intraoperative anesthesia depth monitoring on the prognosis of non-cardiac surgery to guide clinical work.

Methods: Search literature in the database The Cochrane Library, Ovid MEDLINE, EMBASE, Google search \National Knowledge Infrastructure (CNKI), Wanfangdata library and VIP network. We conducted metaanalysis by searching the literature of randomized controlled studies on the effects of different depth of anesthesia monitoring on patients with postoperative delirium and postoperative cognitive dysfunction. The depth of anesthesia monitoring methods include: Bispectral index of EEG, Auditory evoked potential, Primitive EEG monitoring, narcotrend, index of consciousness monitor, entropy, neuroSENSE monitor, SEDline monitor. Two authors independently completed literature retrieval and inclusion, and two authors independently completed literature data collection and bias analysis. The registration number of this study on PROSPERO is CRD42021232899

Results: A total of 7 randomized controlled English literatures with full texts were finally included, with a sample size of 3845 cases. In 5 trials, the depth of anesthesia was monitored by bispectral index (BIS) monitoring, 2 was monitoring of auditory evoked potentials, and 1 was monitoring of raw EEG. They were grouped into monitoring group and control group. Analysis suggests that the depth of anesthesia monitoring, especially the anesthesia guided by BIS monitoring, can reduce the incidence of postoperative delirium. Intraoperative anesthesia depth monitoring reduced the impact of cognitive dysfunction at 1 and 12 weeks after surgery and was statistically significant.

Conclusion: Depth of anesthesia monitoring, especially intraoperative bispectral index monitoring can reduce the occurrence of postoperative delirium in patients undergoing non-cardiac surgery. More clinical trials are needed to evaluate the role of primitive EEG monitoring. Monitoring of the depth of anesthesia is meaningful for reducing postoperative cognitive dysfunction at 1 and 12 weeks after surgery.

\section{Introduction}

Surgery and anesthesia can lead to postoperative delirium and postoperative cognitive dysfunction ${ }^{[1,2]}$. Postoperative delirium is a common complication after major surgery in elderly patients, with an incidence between $15-20 \%{ }^{[3]}$, and is closely related to the prolonged postoperative hospital stay, the prolonged ICU time, and the surge in hospital costs ${ }^{[4,5]}$. Perioperative management, especially surgery and anesthesia ${ }^{[6]}$ factors will affect the incidence of delirium in patients.

Postoperative cognitive dysfunction is a common central nervous system complication after surgery. It usually manifests as cognitive impairment such as memory, mental concentration, impaired language comprehension and decline in social adaptability after surgery. Seriously affect the quality of life of patients. Studies have shown that ${ }^{[7]}{ }^{[8]}$ the incidence of POCD in patients undergoing total hip replacement 7 days and 3 months after surgery is $17 \%$ and $16 \%$, respectively; for patients undergoing 
coronary artery bypass graft surgery, 7 days after surgery The incidence of POCD on the first day and 3 months after the operation was $43 \%$ and $16 \%$, respectively.

There is a certain relationship between anesthesia depth monitoring and postoperative delirium. Whitlock [9] and others did a meta-analysis of 4 studies, comparing BIS monitoring and routine clinical monitoring, the risk ratio of the incidence of postoperative delirium in the two groups was 0.56 (95\% $\mathrm{Cl} 0.42-0.73$ ). Fritz et al. ${ }^{[10]}$ found that low anesthetic concentration and EEG suppression were associated with postoperative delirium in a retrospective cohort study of hip surgery in the elderly. The 2019 study by Wildes et al. is inconsistent with most of the previous conclusions. They believe that intraoperative EEG monitoring cannot reduce the incidence of postoperative delirium.

Compared with postoperative delirium, the depth of anesthesia monitoring has less research on postoperative cognitive dysfunction. Zywiel MG's ${ }^{[11]}$ research on arthroplasty shows that compared with regional anesthesia, the risk of cognitive dysfunction within 7 days after general anesthesia is higher.

There have been systematic reviews and meta-analysis ${ }^{[12 \otimes 13814]}$ on the impact of intraoperative EEG monitoring on postoperative delirium and/or cognitive function, but a comprehensive analysis of various anesthesia depth monitoring methods on postoperative prognosis research system. The evaluation is still relatively small, especially in general anesthesia. Therefore, this study conducted a systematic review and Meta-analysis of randomized controlled trials (RCT) related to this field in order to clarify the impact of intraoperative anesthesia depth monitoring on the prognosis of non-cardiac surgery Perform metaanalysis to guide clinical work》especially on postoperative delirium and postoperative cognitive dysfunction.

\section{Methods}

Our subjects are randomized controlled studies on the effect of intraoperative anesthetic depth monitoring on the prognosis of patients undergoing general anesthesia in non-cardiac surgery. This system review and meta analysis has been registered on PROSPERO, the registration number is 232899 . It will strictly follow Preferred Reporting Items for Systemic Reviews and Meta-analyses (PRISMA) guidelines.

Eligibility criteria: ;The population is general anesthesia patients over 18 years old undergoing noncardiac surgery. Intervention measures: all kinds of anesthetic depth monitoring ,which include: Bispectral

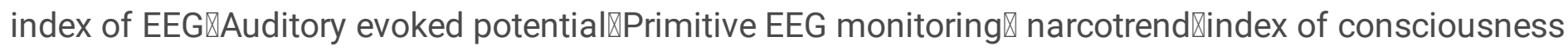
monitor囚entropy囚neuroSENSE monitor》 SEDline monitor. Control measures are general clinical indications to regulate the depth of anesthesia. The mian outcomes are the incidence of postoperative delirium and postoperative cognitive impairment one week and 12 weeks after operation, the second outcomes are the postoperative hospital stay, ICU hospital stay and postoperative mortality.

All the study design are randomized controlled trial,observational designs and nonrandomized trials were excluded. 
Search Strategy : We search literature in the English databases including the Cochrane Library, pubmed,

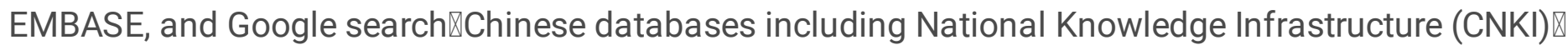
Wanfangdata library and VIP network. English search terms: postoperative delirium, postoperative cognitive dysfunction, the depth of anesthesia, electroencephalography, processed electroencephalogram, electroencephalography-guided, bispectral index, BIS, audityevoked potential index (AAI), AEP, narcotrend, CSI, index of consciousness monitor, entropy, neuroSENSE monitor , SEDline monitor, a randomised controlled trial, etc. The search time is set from 1990 to February 1, 2021, because EEG monitoring was only used after 1990. The search strategy is formulated in accordance with the PICOS principles, such as PubMed search strategy:

Study selection and Quality assement

Document screening and data collection are cbined with search results to eliminate identical documents. Read the title and abstract of the literature for a preliminary screening, exclude non-related literature, and then read the full text to find research materials that meet the standards. The data table extracts data, including recording basic information (author, nationality, number of cases, sex ratio, age distribution). The data collection and analysis are completed by two authors independently and checked with each other. If there is any different views, it will be resolved through consultation.

Risk of Bias in Included Studies Quality evaluation adopts the bias risk assessment form of the Review manager 5.3 conducts bias risk assessment on references. The evaluation criteria include: whether to group randomly, whether to double-blind, whether there are incomplete data records (such as whether there are data descriptions of withdrawal from the group or lost to follow-up due to various reasons), and whether there is no selective report of results (such as whether there are obvious results or data The description is missing, missing), whether there is no other bias. "Green" means low risk, "yellow" means uncertain, and "red" means high risk; then draw a bias risk map. Sensitivity testing is done by reanalyzing the data by changing the statistical analysis model.

Data Extraction and Management Two authors independently read the included literature, extract data, and resolve different opinions through consultation. The details of the data include the time the article was published, the author, the characteristics of the included population, the method of anesthesia, the method of surgery, the number of non-cardiac surgery, intervention measures, the number of patients with postoperative delirium and postoperative cognitive dysfunction, the number of hospitalization days, ICU Length of hospitalization and number of deaths, etc.

Data Synthesis:Statistical methods Use Review Manager 5.3 software for statistical analysis. Count data uses Risk ratio (RR) and its $95 \% \mathrm{Cl}$ as the effect size to express the result. The measurement data calculates the mean difference (MD) to indicate the size of the effect. Analyze the heterogeneity of the included studies, $\mathrm{P}<0.05$ indicates significant heterogeneity, and $\mathrm{I}^{2}$ quantitatively describes the heterogeneity difference. If the heterogeneity is significant $\left(I^{2} \geq 50 \%\right)$, a random effects model is used for Meta -analysis. If $\mathrm{I}^{2}<50 \%$, use random effects model or fixed effects model. 
Sensitivity Analysis

Sensitivity studies are used to analyze the impact of a single study on the heterogeneity of the overall study. We exclude them one by one for each trial, recalculate the aggregated relative riskfor the remaining trials using the fixed effects model.

\section{Results}

Study selection: The flow chart of literature search and screening is as follows: the total number of documents we retrieved is 1085 , and finally there are 7 included documents that meet the criteria.

Study Characteristics: Included documents: 7 English documents with full texts were finally included in the above table (Table 1). All 7 articles are randomized controlled studies. The total sample size included was 3845 cases. The depth of anesthesia monitoring in 5 trials was BIS monitoring, 2 were AAI monitoring, and 1 was raw EEG monitoring. In data analysis, in order to unify the grouping, we unified into the monitoring group and the control group.

Risk of Bias in Included Studies

Figure 3 and Figure 4 are the bias of each study and the bias of all studies, respectively.

Primary Analysis Our primary result is the impact of intraoperative anesthesia depth monitoring on postoperative delirium and postoperative cognitive function.

For comparison of the incidence of postoperative delirium, a total of 5 articles were included, 3 articles used BIS monitoring, 1 article used auditory evoked potentials, and 1 article used original EEG monitoring. There were 2924 patients in total, see Figure $5, I^{2}=75 \%, p=0.003$. $(O R=0.77,95 \% \mathrm{Cl} 0.64$ to $0.93, P=$ $0.003)$, the incidence of postoperative delirium in the monitoring group was lower than that in the control group.

Three articles related to the analysis of the incidence of postoperative delirium by BIS monitoring, $I^{2}=0$, $p=0.57$. As shown in Figure 6, there are a total of 2138 patients, and the incidence of postoperative delirium in the monitoring group is lower than that in the control group $(\mathrm{OR}=0.65,95 \% \mathrm{Cl} 0.53 \otimes 0.81, \mathrm{P}=$ 0.0001)

The influence of intraoperative anesthesia depth monitoring on cognitive dysfunction one week after surgery is shown in Figure 7. A total of 5 articles were included, with 2471 patients, as shown in Figure 7, $\mathrm{Q}=10.23 \otimes \mathrm{I}^{2}=61 \%, \mathrm{p}=0.04 .(\mathrm{OR}=0.75,95 \% \mathrm{Cl} 0.60 \otimes 0.94, \mathrm{P}<0.05)$ The incidence of cognitive dysfunction in the monitoring group was lower than that in the control group one week after surgery.

The effect of intraoperative anesthesia depth monitoring on cognitive dysfunction at 12 weeks after surgery is shown in Figure 8. A total of three studies were included in the study, shown in Figure 8 . There were a total of 2051 patients in the monitoring group and the control group. There was no significant 
heterogeneity between the data and the data $\left(Q=0.14, P=0.93, I^{2}=0\right)$. The incidence of cognitive dysfunction in the monitoring group was lower than the control group at 12 weeks after surgery ( OR $=0.69,95 \% \mathrm{Cl} 0.49 \otimes 0.96, \mathrm{P}<0.05)$.

Secondary Outcomes

Two literatures made statistics and analysis on the time from postoperative to discharge of patients. Random effects model will be included in the meta-analysis. The results indicate that the difference between the two groups is statistically significant (Pख0.00001, MD: $-1.00,95 \% \mathrm{Cl}:-1.11 \sim-0.88$ ). As Figure 9 .

Only one article by Chan has statistics on the length of ICU stay, and there is no statistical difference in the length of ICU stay between the two groups.

There are 2 literatures on the total mortality rate, 1605 patients. The postoperative mortality rate is statistically analyzed. The study of Jildestal 2011 is the mortality rate of one year after operation, and the study of Radtke is the mortality rate of 3 months after operation. Combining all mortality rates as the total mortality rate, Wildes' study did not include statistics because it did not find data on patients with non-heart diseases. The random effects model was used for meta-analysis, and the results showed that the depth of anesthesia monitoring had no effect on the postoperative mortality of patients $(P=0.97, R R$ : 1.01, 95\%Cl: 0.62-1.64).

Sensitivity Analysis

The Wildes study was deleted, and the remaining 4 papers were subjected to meta-analysis, $I^{2}=29 \%$, $p=0.24$. Figure 5 shows that the source of heterogeneity is Wilds' research. A total of 2170 patients, the incidence of postoperative delirium in the monitoring group was lower than that in the control group (OR $=0.64,95 \% \mathrm{Cl} 0.53 \varangle 0.79, \mathrm{P}<0.0001)$

\section{Discussion}

Postoperative delirium is a common complication 1-3 days after operation, especially for elderly patients, which directly leads to prolonged postoperative hospital stay and increased postoperative mortality [22-24]. Postoperative cognitive impairment is a common complication after anesthetic surgery. Perioperative factors, such as age, sex, preoperative medication, preoperative basic diseases, preoperative education, alcoholism, type of operation, anesthetic medication, depth of anesthesia, postoperative infection, etc., can form or promote the occurrence of POCD ${ }^{[25]}$.

The meta-analysis on the impact of delirium included 5 randomized controlled studies of depth of anesthesia monitoring. All combined data analysis showed that there was a significant heterogeneity between the studies $I^{2}=75 \%$. The results suggest that depth of anesthesia monitoring can reduce the incidence of postoperative delirium. $(P<0.05)$. 
Meta-analysis of 3 BIS monitoring studies, a total of 2138 patients, the incidence of postoperative delirium in the monitoring group was lower than that in the control group $(\mathrm{OR}=0.65,95 \% \mathrm{Cl} 0.53$ to 0.81 , $P=0.0001)$. Sensitivity research suggests that the source of heterogeneity is Wildes' research. Sensitivity analysis suggests that the source of heterogeneity is Wildes's study, because only this article is about raw EEG monitoring. This study included a total of 1232 patients and was the first large-sample study on raw EEG monitoring. The study suggested that the incidence of postoperative delirium in the EEG monitoring group was not statistically different from that in the control group on the 1-5 days after surgery $(P=0.22)$, indicating that the original EEG monitoring could not reduce the incidence of postoperative delirium in elderly patients. The theoretical basis of this study is based on the assumption that explosive suppression in BIS monitoring is related to postoperative delirium ${ }^{[10]}$, but in the course of the experiment, the requirements for controlling the depth of anesthesia in this study are more stringent than pure BIS monitoring. Therefore, the impact of original EEG monitoring on postoperative delirium is worthy of further study: According to the visual analysis of original EEG, whether the burst inhibition ratio can be reduced and the occurrence of postoperative delirium may be the direction of future research.

The reason why the depth of anesthesia monitoring reduces delirium is not clear. BIS monitoring is related to reducing the exposure of anesthetics, especially the reduction of extreme low BIS values $(B I S<20)^{[19]}$, thus changing the anesthesia management methods including intraoperative neuromonitoring, thereby Reduce postoperative delirium. There is a saying that avoiding explosive suppression of intraoperative EEG can reduce postoperative delirium, because explosive suppression of EEG may mean unnecessary deep anesthesia ${ }^{[26]}$. Of course, EEG of explosive suppression also appears in patients with coma and traumatic brain injury [27]. A retrospective study of 618 samples of Fritz et al. found that patients with explosive suppression of EEG under low-concentration inhalation anesthesia were more likely to develop delirium after surgery, which also proved the connection between anesthetic exposure and postoperative delirium ${ }^{[10]}$.

Jldenstal's two studies are both about auditory evoked potentials. He believes that auditory evoked potentials to monitor the depth of anesthesia can reduce the amount of anesthetics, reduce fluid requirements and the use of vasoactive drugs, thereby enhancing cardiovascular stability and ultimately reducing The incidence of cognitive dysfunction in early postoperative patients. Chan believes that the occurrence of POCD at 12 weeks after surgery is a continuation of postoperative delirium, so the risk factors of the two are also similar, including: high-dose anesthetics, low average BIS during surgery, and long-term deep anesthesia (BIS< 40) and aging factors ${ }^{[2]}$. Therefore, reducing the dosage of anesthetics through BIS monitoring and avoiding deep anesthesia may be the reason why the depth of anesthesia monitoring reduces cognitive dysfunction within 12 weeks after surgery. At present, there are ongoing studies trying to clarify whether postoperative cognitive dysfunction is one of the results of postoperative delirium ${ }^{[28]}$, and the exact relationship between them needs further observation ${ }^{[29]}$.

The meta-analysis included fewer studies on postoperative hospital stay and ICU hospital stay and total mortality. It only suggested that the monitoring group had less postoperative hospital stay, and there was 
no statistical difference in postoperative total mortality between the two groups. However, there is already strong evidence supporting that the occurrence of postoperative delirium will greatly extend the length of hospital stay ${ }^{[30]}$, so it is still necessary to actively prevent the occurrence of postoperative delirium.

In contrast to previous meta-analysis of this type, the included population in this article is over 18 years old. In terms of anesthesia methods, studies on sedation under intraspinal anesthesia are excluded. In terms of monitoring methods, it involves various current clinical methods of monitoring the depth of anesthesia ${ }^{[31]}$. This study included two documents using auditory evoked potential monitoring, but the sample size was not large. The object of this study is also different from the articles of Yi Sun et al ${ }^{[14]}$, excluding cardiac surgery and reducing bias.

The research results suggest that intraoperative anesthesia depth monitoring can reduce the occurrence of delirium after non-cardiac surgery, and BIS monitoring can reduce the incidence of cognitive dysfunction in one week and 12 weeks after surgery. The research conclusion and Kristen K MacKenzie ${ }^{[13]}$ and Punjasawadwong $Y^{[12]}$ Similar to others. Compared with previous meta studies, the biggest highlight of this article is the inclusion of the original EEG monitoring research published in 2019. The effect of original EEG monitoring on postoperative delirium and even cognitive dysfunction deserves further study. The shortcomings of this study are due to the limitation of the included literature. There is no further analysis of the influence of different general anesthesia methods such as intravenous anesthesia or inhalation anesthesia on the conclusion, and no further meta-analysis of the specific BIS value.

\section{Conclusion}

Intraoperative BIS monitoring can reduce the incidence of postoperative delirium in patients, and more clinical trials are needed for primitive EEG monitoring. Monitoring the depth of anesthesia is meaningful for reducing postoperative cognitive dysfunction in one week and 12 weeks after surgery.

\section{Declaration}

Acknowledgements No acknowledgements.

Funding No funding was received for this study.

Data Availability The data sets generated and analysed during the current study are available from the corresponding author on reasonable request.

Ethics approval and consent to participate Not applicable.

Consent for publication Not applicable.

Competing interests The authors declare no competing interests. 


\section{References}

1. Inouye SK, Westendorp RGJ, Saczynski JS. Delirium in elderly people. The Lancet. 2014; 383(9920): 911-922.

2. Chan MTV, Cheng BCP, Lee TMC. BIS-guided anesthesia decreases postoperative delirium and cognitive decline. J Neuro Anesthesiol. 2013; 25(1): 33-42.

3. Newman S, et al. Postoperative cognitive dysfunction after noncardiac surgery: a systematic review. J Am Soc Anesthesiol. 2007; 106(3): 572-590.

4. Gottesman RF, et al. Delirium after coronary artery bypass graft surgery and late mortality. Ann Neurol. 2010; 67(3): 338-344.

5. Franco K, et al. The cost of delirium in the surgical patient. Psychosomatics. 2001; 42(1): 68-73.

6. Steiner LA. Postoperative delirium. Part 1: pathophysiology and risk factors. Eur J Anaesthesiol (EJA). 2011; 28(9): 628-636.

7. Evered L, et al. Postoperative cognitive dysfunction is independent of type of surgery and anesthetic. Anesthesia \& Analgesia. 2011; 112(5): 1179-1185.

8. Paredes $\mathrm{S}$, et al. Post-operative cognitive dysfunction at 3 months in adults after non-cardiac surgery: a qualitative systematic review. Acta Anaesthesiologica Scandinavica; 2016; 60(8): 10431058.

9. Whitlock EL, et al. Postoperative delirium in a substudy of cardiothoracic surgical patients in the BAG-RECALL clinical trial. Anesthesia and Analgesia. 2014; 118(4): 809.

10. Fritz B A, et al. Intraoperative electroencephalogram suppression predicts postoperative delirium. Anesthesia and Analgesia. 2016; 122(1): 234.

11. Zywiel MG, et al. The influence of anesthesia and pain management on cognitive dysfunction after joint arthroplasty: a systematic review. Clin Ortho Related Res. 2014; 472(5): 1453-1466.

12. Punjasawadwong $Y$, et al. Processed electroencephalogram and evoked potential techniques for amelioration of postoperative delirium and cognitive dysfunction following non-cardiac and nonneurosurgical procedures in adults. Cochrane Database of Systematic Reviews. 2018; (5): CD011283.

13. MacKenzie KK, et al. Processed electroencephalogram monitoring and postoperative delirium: a systematic review and meta-analysis. Anesthesiology. 2018; 129(3): 417-427.

14. Sun $Y$, et al. Electroencephalography-guided anesthetic delivery for preventing postoperative delirium in adults: an updated meta-analysis. Anesthesia \& Analgesia. 2020; 131(3): 712-719.

15. Jildenstål PK, et al. Effect of auditory evoked potential-guided anaesthesia on consumption of anaesthetics and early postoperative cognitive dysfunction: a randomised controlled trial. Eur $\mathrm{J}$ Anaesthesiol (EJA). 2011; 28(3): 213-219.

16. Jildenstål PK, et al. AAl-guided anaesthesia is associated with lower incidence of 24-h MMSE< 25 and may impact the IL-6 response. Int J Surg. 2014; 12(4): 290-295. 
17. Ballard C, et al. Optimised anaesthesia to reduce post operative cognitive decline (POCD) in older patients undergoing elective surgery, a randomised controlled trial. PloS One. 2012; 7(6): e37410.

18. Radtke FM, et al. Monitoring depth of anaesthesia in a randomized trial decreases the rate of postoperative delirium but not postoperative cognitive dysfunction. Bri J Anaesthesia. 2013; 110(suppl_1): i98-i105.

19. Chan MTV, et al. BIS-guided anesthesia decreases postoperative delirium and cognitive decline. J Neuro Anesthesiol. 2013; 25(1): 33-42.

20. Zhou Y, Li Y, Wang K. Bispectral index monitoring during anesthesia promotes early postoperative recovery of cognitive function and reduces acute delirium in elderly patients with colon carcinoma: A prospective controlled study using the attention network test. Medical science monitor: international medical journal of experimental and clinical research. 2018; 24: 7785.

21. Wildes TS, et al. Effect of electroencephalography-guided anesthetic administration on postoperative delirium among older adults undergoing major surgery: the ENGAGES randomized clinical trial. Jama. 2019; 321(5): 473-483.

22. Demeure MJ, Fain MJ. The elderly surgical patient and postoperative delirium. J Am Col Surg. 2006; 203(5): 752-757.

23. Leslie DL, et al. One-year health care costs associated with delirium in the elderly population. Arch Int Med. 2008; 168(1): 27-32.

24. Crocker $E$, et al. Long-term effects of postoperative delirium in patients undergoing cardiac operation: a systematic review. Ann Thora Surg. 2016; 102(4): 1391-1399.

25. Moller JT, et al. Long-term postoperative cognitive dysfunction in the elderly: ISPOCD1 study. The Lancet. 1998; 351(9106): 857-861.

26. Hagihira S. Changes in the electroencephalogram during anaesthesia and their physiological basis. Bri J Anaesthesia. 2015; 115(suppl_1): i27-i31.

27. Brown EN, Lydic R, Schiff ND. General anesthesia, sleep, and coma. N Eng J Med. 2010; 363(27): 2638-2650.

28. Aranake-Chrisinger $A$, et al. Ability of postoperative delirium to predict intermediate-term postoperative cognitive function in patients undergoing elective surgery at an academic medical centre: protocol for a prospective cohort study. BMJ Open. 2018; 8(3): e017079.

29. Cascella $M$, et al. Postoperative delirium and postoperative cognitive dysfunction: updates in pathophysiology, potential translational approaches to clinical practice and further research perspectives. Minerva Anestesiologica. 2017; 84(2): 246-260.

30. Sessler DI, et al. Hospital stay and mortality are increased in patients having a "triple low" of low blood pressure, low bispectral index, and low minimum alveolar concentration of volatile anesthesia. J Am Soc Anesthesiologists. 2012; 116(6): 1195-1203.

31. Fahy BG, Chau DF. The technology of processed electroencephalogram monitoring devices for assessment of depth of anesthesia. Anesthesia \& Analgesia. 2018; 126(1): 111-117. 


\section{Table}

Table 1 is available in the supplementary Files

\section{Figures}

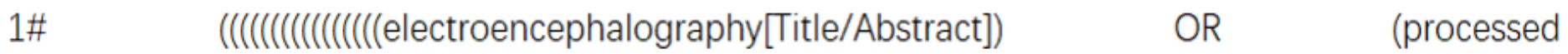
electroencephalogram[Title/Abstract])) OR (electro?encephalograph*[Title/Abstract])) OR (electroencephalography-guided[Title/Abstract])) OR (EEG[Title/Abstract])) OR (bispectral index[Title/Abstract])) OR (BIS[Title/Abstract])) OR (anesthesia depth[Title/Abstract])) OR (the depth of anesthesia[Title/Abstract])) OR (AEP[Title/Abstract])) OR (AAl[Title/Abstract])) OR (auditory evoked potential index[Title/Abstract])) OR (narcotrend[Title/Abstract])) OR (index of consciousness monitor[Title/Abstract])) OR (entropy[Title/Abstract])) OR (neuroSENSE monitor[Title/Abstract])) OR (SEDline monitor[Title/Abstract])

2\# ((((POD[Title/Abstract]) OR (POCD[Title/Abstract])) OR ( confusional state*[Title/Abstract])) OR (congnitive disorder[Title/Abstract]))

3\# (cognitive dysfunction) OR ( delirium[ MeSH Terms])

4\# (((randomized controlled trial[Title/Abstract]) OR (controlled[Title/Abstract])) OR (randomized[Title/Abstract])) OR (trial[Title/Abstract])

$5 \#$ 1\# and $2 \#$ and $3 \#$ and $4 \#$

\section{Figure 1}

PubMed search strategy 
Databases searched:0vid

MEDLINE ( $n=443)$, PubMled ( $n=435)$, EMBASE

( $n=32)$, Cochrane Library $(n=16)$, and Google search $(n=115)$, CKKI ( $n=19)$ hinfangdata

library $(n=16)$ and VIP network $(n=9)$

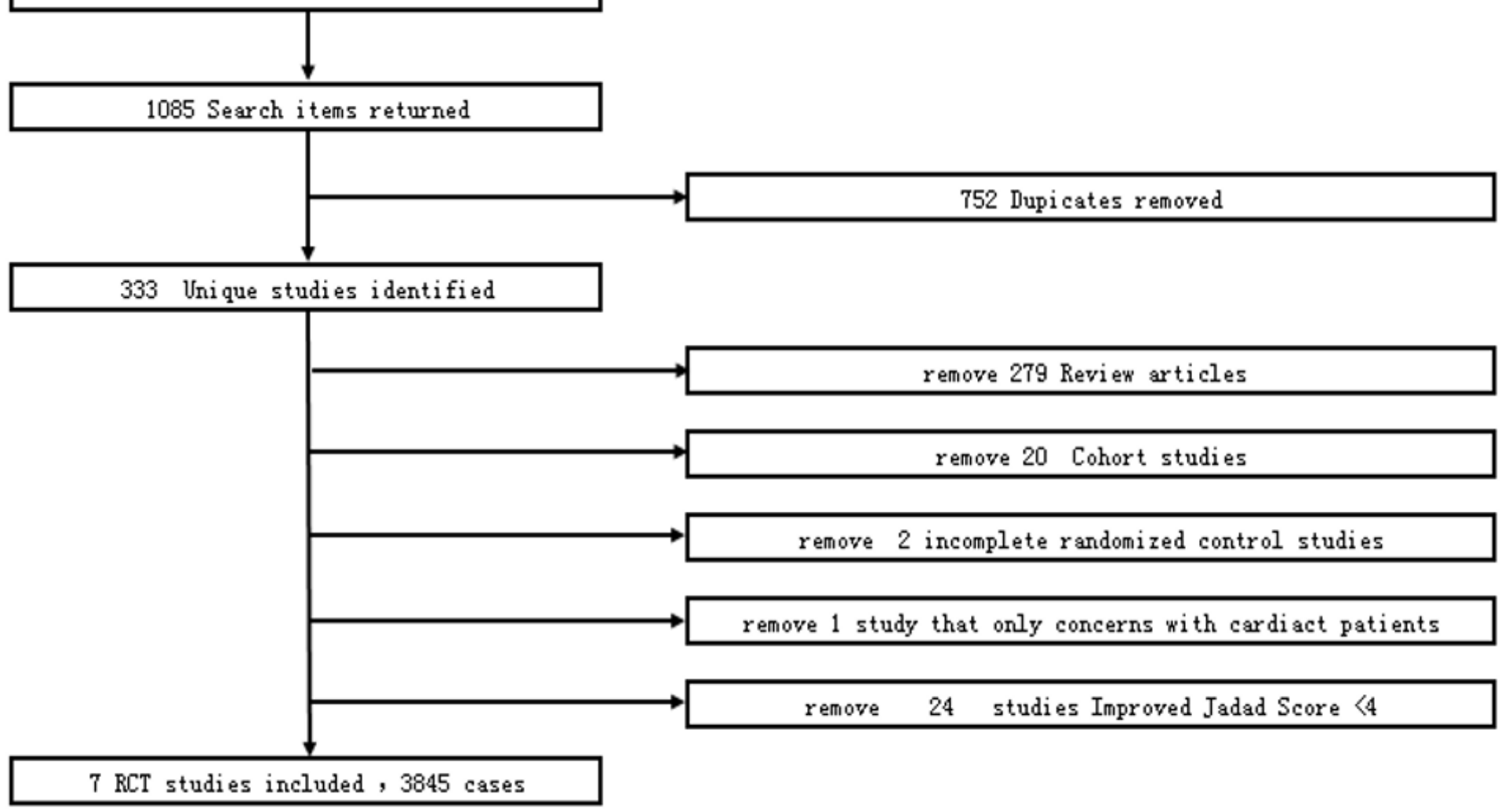

\section{Figure 2}

Study Flow Diagram 


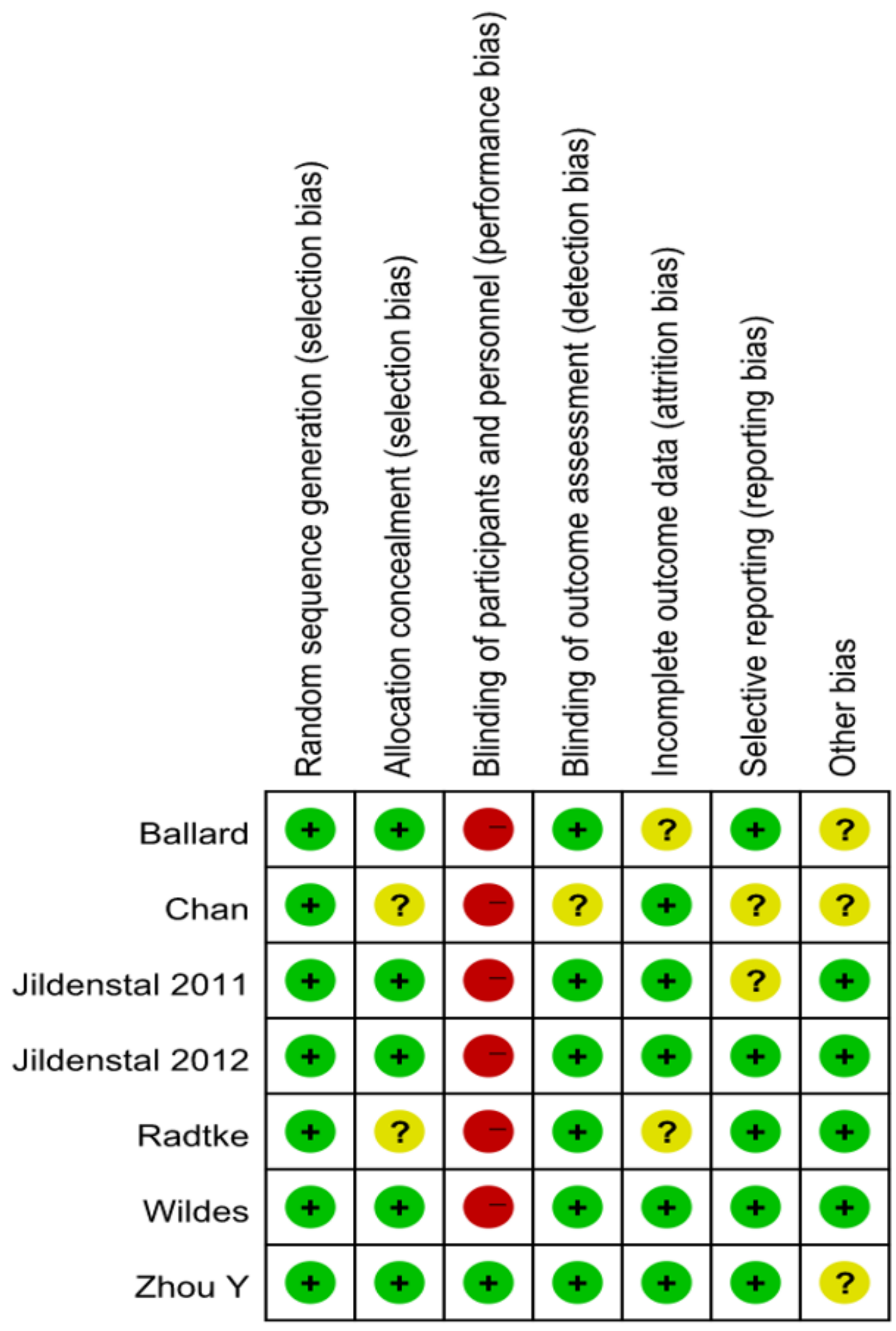

Figure 3

Risk of bias for each study. 
Random sequence generation (selection bias)

Allocation concealment (selection bias)

Blinding of participants and personnel (performance bias)

Blinding of outcome assessment (detection bias)

Incomplete outcome data (attrition bias)

Selective reporting (reporting bias)

Other bias
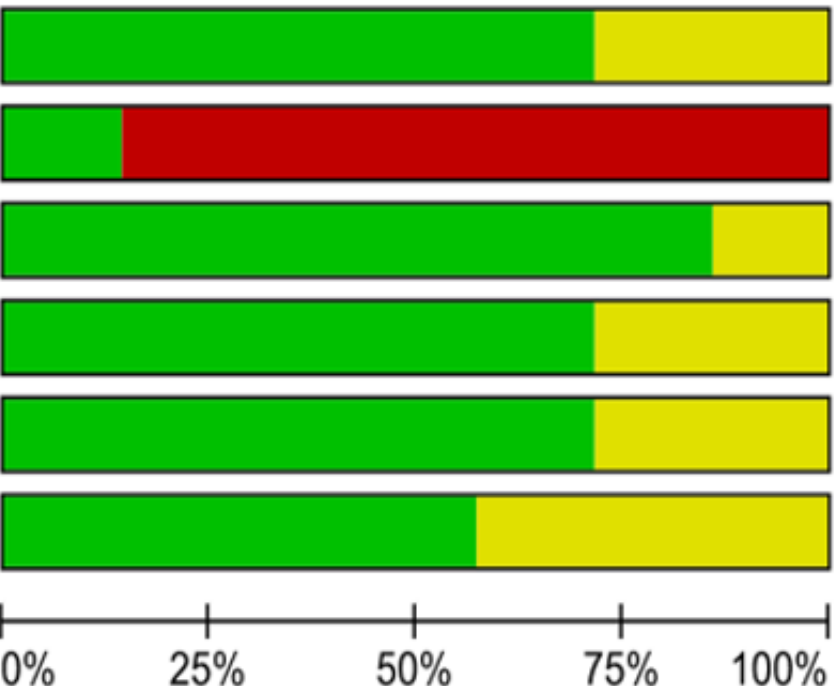

\section{$\square$ Low risk of bias}

\section{Figure 4}

Risks of bias summary.

\begin{tabular}{|c|c|c|c|c|c|c|}
\hline \multirow[b]{2}{*}{ Study or Subgroup } & \multicolumn{2}{|c|}{ Monitored care } & \multicolumn{2}{|c|}{ Routine care } & \multirow[b]{2}{*}{ Weight } & \multirow{2}{*}{$\begin{array}{c}\text { Risk Ratio } \\
\mathrm{M}-\mathrm{H}, \text { Random, } 95 \% \mathrm{Cl}\end{array}$} \\
\hline & Events & Total & Events & Total & & \\
\hline Chan & 70 & 450 & 109 & 452 & $34.8 \%$ & $0.58[0.42,0.81]$ \\
\hline Jildestal 2012 & 1 & 16 & 7 & 16 & $2.5 \%$ & $0.09[0.01,0.82]$ \\
\hline Radtke & 95 & 575 & 124 & 580 & $39.1 \%$ & $0.73[0.54,0.98]$ \\
\hline Wildes & 85 & 374 & 69 & 380 & $20.1 \%$ & $1.33[0.93,1.89]$ \\
\hline Zhou Y & 7 & 41 & 11 & 40 & $3.5 \%$ & $0.54[0.19,1.58]$ \\
\hline Total (95\%Cl) & & 1456 & & 1468 & $100.0 \%$ & $0.77[0.64,0.93]$ \\
\hline
\end{tabular}

$\begin{array}{cc}\text { Total events } & 258 \\ \text { Heterogeneity: } \mathrm{Chi}^{2}=15.93, \mathrm{df}=4(\mathrm{P}=0.003) ; \mathrm{I}^{2}=75 \%\end{array}$

320

Test for overall effect: $Z=2.76(P=0.006)$

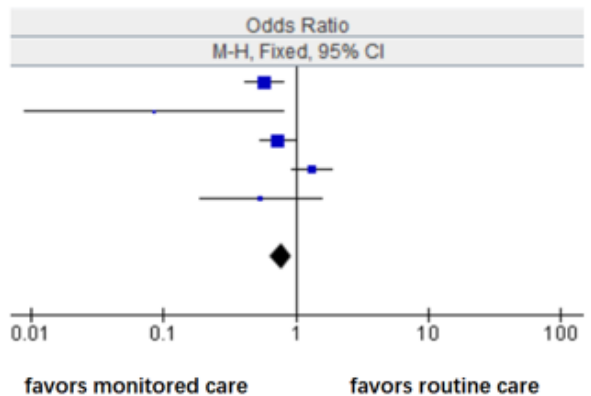

\section{Figure 5}

Forest plot of postoperative delirium in randomized controlled trials. Analysis of the incidence of postoperative delirium by monitoring the depth of anesthesia

\begin{tabular}{|c|c|c|c|c|c|c|}
\hline \multirow[b]{2}{*}{ Study or Subgroup } & \multicolumn{2}{|c|}{ Monitored care } & \multicolumn{2}{|c|}{ Routine care } & \multirow[b]{2}{*}{ Weight } & \multirow{2}{*}{$\begin{array}{c}\text { Risk Ratio } \\
\text { M-H,Random, } 95\end{array}$} \\
\hline & Events & Total & Events & Total & & \\
\hline Chan & 70 & 450 & 109 & 452 & $26.3 \%$ & $0.58[0.42,0.81]$ \\
\hline Radtke & 95 & 575 & 124 & 580 & $29.5 \%$ & $0.73[0.54,0.98]$ \\
\hline Zhou Y & 7 & 41 & 11 & 40 & $2.6 \%$ & $0.54[0.19,1.58]$ \\
\hline Total $(97 \% \mathrm{Cl})$ & & 1066 & & 1072 & $100.0 \%$ & $0.65[0.53,0.81]$ \\
\hline Total events & 172 & & 244 & & & \\
\hline \multicolumn{7}{|c|}{ Heterogeneity: $\mathrm{Chi}^{2}=1.12, \mathrm{df}=2(\mathrm{P}=0.57) ; \mathrm{I}^{2}=0 \%$} \\
\hline \multicolumn{7}{|c|}{ Test for overall effect: $Z=3.86(P=0.0001)$} \\
\hline
\end{tabular}

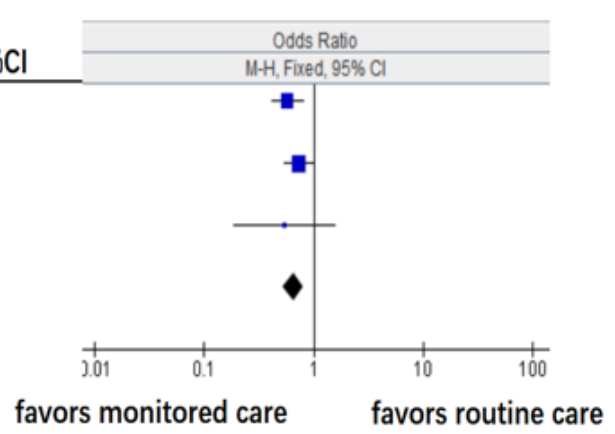


Figure 6

Forest plot of postoperative delirium in randomized controlled trials. Analysis of BIS monitoring on the incidence of postoperative delirium

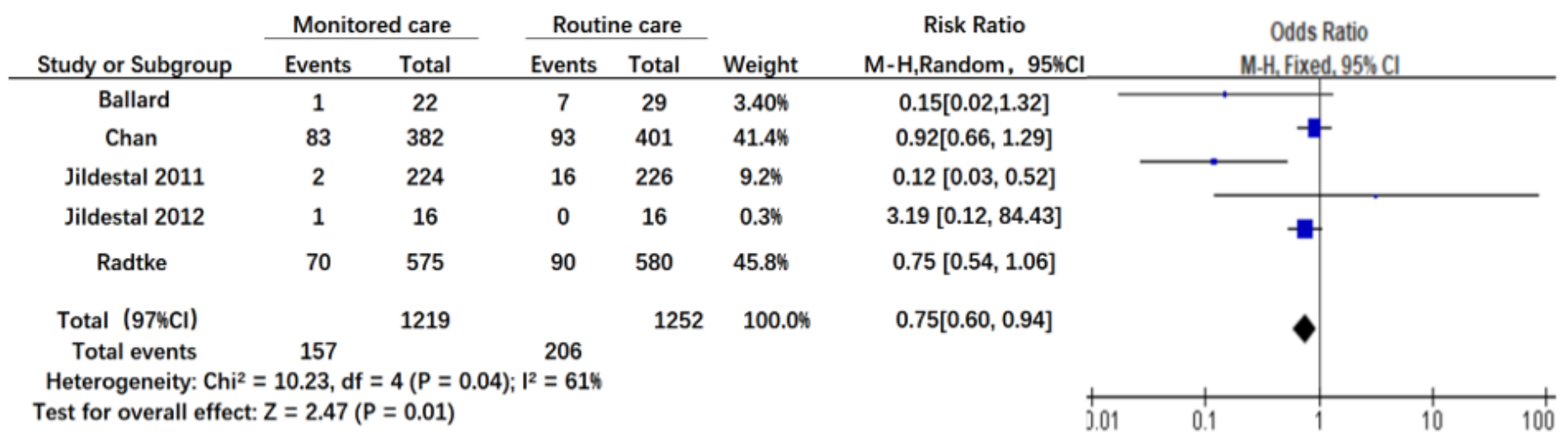

favors monitored care favors routine care

\section{Figure 7}

Forest plot of postoperative cognitive function in randomized controlled trials. Analysis of the incidence of cognitive dysfunction in the depth of anesthesia monitoring one week after surgery.

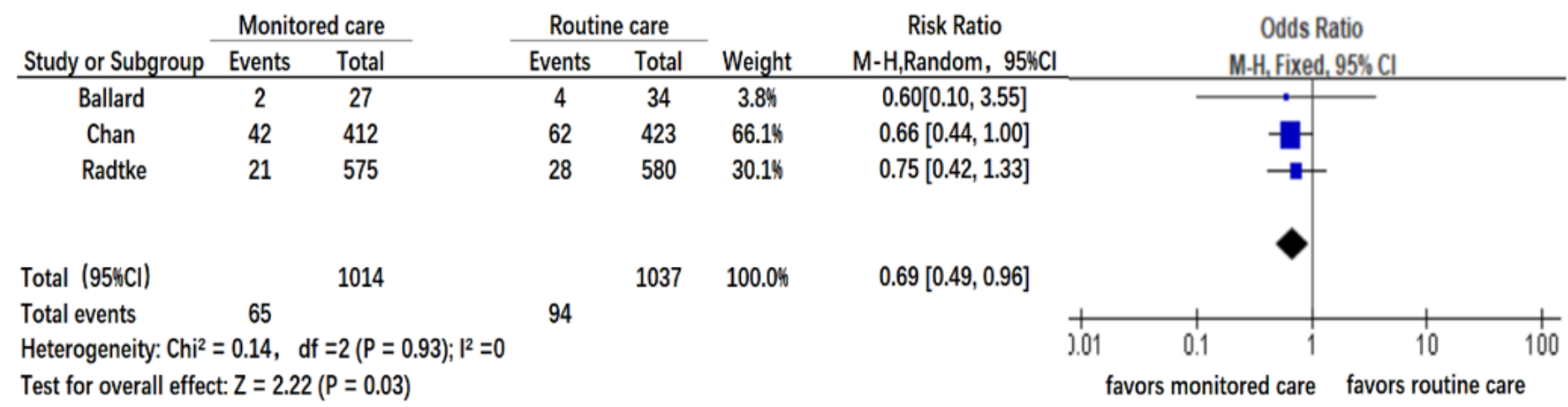

\section{Figure 8}

Forest plot of postoperative cognitive function in randomized controlled trials. Analysis of the incidence of cognitive dysfunction in the depth of anesthesia monitoring four week after surgery.

\begin{tabular}{|c|c|c|c|c|c|c|c|}
\hline \multirow[b]{2}{*}{ Study or Subgroup } & \multicolumn{3}{|c|}{ Monitored care } & \multicolumn{3}{|c|}{ Routine care } & \multirow[t]{2}{*}{ Weight $\%$} \\
\hline & Mean & SD & Sum & Mean & SD & Sum & \\
\hline Chan & 7 & 0.8 & 450 & 8 & 1 & 452 & $99.60 \%$ \\
\hline Radtke & 15.7 & 16.9 & 575 & 15.9 & 14.6 & 580 & $0.40 \%$ \\
\hline Total $(95 \% \mathrm{Cl})$ & & & 1025 & & & 1032 & \\
\hline
\end{tabular}

Heterogeneity: $\mathrm{Chi}^{2}=0.74, \mathrm{df}=1(\mathrm{P}=0.39) ; \mathrm{I}^{2}=0 \%$

Test for overall effect: $Z=16.57(P<0.00001)$ 
The effect of depth of anesthesia monitoring on postoperative hospital stay

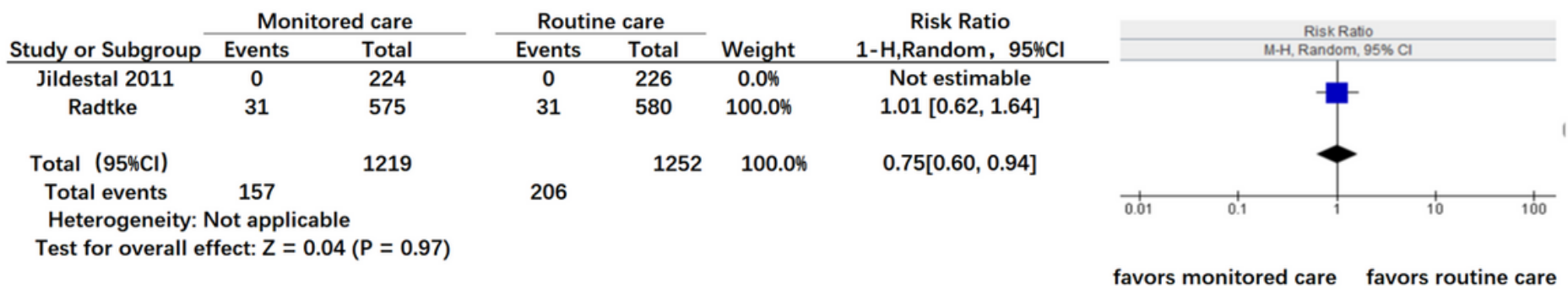

\section{Figure 10}

The effect of monitoring the depth of anesthesia on the postoperative total mortality

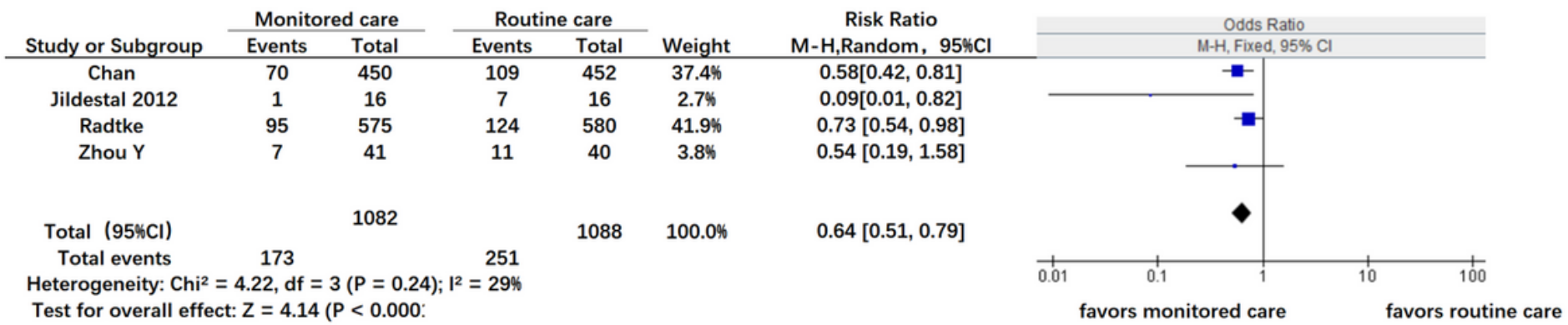

\section{Figure 11}

Sensitivity analysis of depth of anesthesia monitoring on the incidence of postoperative delirium

\section{Supplementary Files}

This is a list of supplementary files associated with this preprint. Click to download.

- Table1.png 